\title{
Dynamic coherent beam combining based on a setup of microlens arrays
}

Prossotowicz, Maike, Heimes, Andreas, Flamm, Daniel, Jansen, Florian, Otto, Hans-Jürgen, et al.

Maike Prossotowicz, Andreas Heimes, Daniel Flamm, Florian Jansen, HansJürgen Otto, Aleksander Budnicki, Uwe Morgner, Alexander Killi, "Dynamic coherent beam combining based on a setup of microlens arrays," Proc. SPIE 11266, Laser Resonators, Microresonators, and Beam Control XXII, 1126612 (2 March 2020); doi: 10.1117/12.2547303

SPIE. Event: SPIE LASE, 2020, San Francisco, California, United States 


\title{
Dynamic coherent beam combining based on a setup of microlens arrays
}

\author{
Maike Prossotowicz, ${ }^{a}$ Andreas Heimes, ${ }^{b}$ Daniel Flamm, ${ }^{b}$ Florian Jansen, ${ }^{a}$ Hans-Jürgen Otto,${ }^{a}$ \\ Aleksander Budnicki, ${ }^{a}$ Uwe Morgner ${ }^{c}$ and Alexander Killi ${ }^{a}$ \\ ${ }^{a}$ TRUMPF Laser GmbH, Aichhalder Str. 39, 78713 Schramberg, Germany \\ ${ }^{b}$ TRUMPF Laser- und Systemtechnik GmbH, Johann-Maus-Str. 2, 71254 Ditzingen, Germany \\ ${ }^{c}$ Institut für Quantenoptik, Leibniz-Universität Hannover, Welfengarten 1, 30167 Hannover, \\ Germany
}

\begin{abstract}
A novel optical concept is introduced with standard components for highly efficient coherent beam combining a system of $(N \times N)$ beams. In a proof-of-principle experiment a well-defined setup with microlens arrays (MLAs) is used to create a beam matrix of $5 \times 5$ beams. For the combination step the same setup is employed, and the created 25 beams are combined. A combination efficiency above $90 \%$ is achieved. Furthermore, the concept allows for dynamic beam combination, i.e., the resulting number of beams and corresponding positions can be controlled by the absolute phases of the array of input beams. A proof-of-principle experiment shows excellent agreement with the model.
\end{abstract}

Keywords: Coherent beam combination, Ultrashort pulses, Ultrafast optics, Beam steering, Beam shaping, Microlens arrays

\section{INTRODUCTION}

In industry and science high intensity laser pulses have found many applications. ${ }^{1}$ Regardless of the laser amplifier architecture, intrinsic limits hinder scaling parameters. Coherent beam combination $(\mathrm{CBC})^{2}$ represents the most promising concept for further power and energy scaling of ultrafast laser sources. ${ }^{3}$ With CBC average powers for ultrafast lasers of $3.5 \mathrm{~kW}^{4}$ and pulse energies up to $12 \mathrm{~mJ}^{5}$ can be achieved. This offers a new possibility for scientific and industrial applications such as material processing. ${ }^{6-10}$

A CBC system is an interferometer with amplifiers in the interferometer arms. The pulses of the common seed source are subsequently split and sent into the interferometer arms, amplified and combined into a single output beam. Through e.g. atmospheric turbulences, vibrations or thermal effects optical path length differences between the pulses occur. Therefore, an active stabilization technique with a phase detector and a phase modulator is necessary. The phase difference between the pulses can be determined directly or derived by dithering the phases and measuring the impact of these changes on the output signal. A direct phase detection technique is a Hänsch-Coillaud $(\mathrm{HC})^{11}$ detector. Indirect detection techniques are locking of optical coherence by singledetector electronic-frequency tagging technology (LOCSET) ${ }^{12}$ or Stochastic Parallel Gradient Descent (SPGD) ${ }^{13}$ technique. The phase information of the phase detector is used as error signal to change the path length differences between the channels. This is realized with phase modulators in at least $N-1$ channels, for example with a mirror mounted on a piezo electric actuator, a piezo-electric fiber stretcher or an spatial light modulator (SLM). The combination efficiency of such a system depends on how well the path length differences can be actively stabilized and on the combination element itself. The basic principle of a CBC system is shown in Fig. 1. The combination element is of particular importance in a CBC system, since it has to combine separated beams into one beam while preserving the beam quality and keeping losses as low as possible. In addition, it has to endure a high average power and pulse energy. Therefore, different elements have been successfully proposed for ultrafast lasers such as partially reflective surfaces, ${ }^{14}$ polarization dependent beam splitters ${ }^{15}$ or segmented beam mirrors. ${ }^{16}$ In this paper a proof of principle experiment with two microlens arrays (MLA) as splitter and

Further author information:

E-Mail: daniel.flamm@trumpf.com.

Laser Resonators, Microresonators, and Beam Control XXII, edited by Alexis V. Kudryashov,

Alan H. Paxton, Vladimir S. Ilchenko, Andrea M. Armani, Proc. of SPIE Vol. 11266, 1126612

(C) 2020 SPIE · CCC code: $0277-786$ X/20/\$21 · doi: 10.1117/12.2547303 


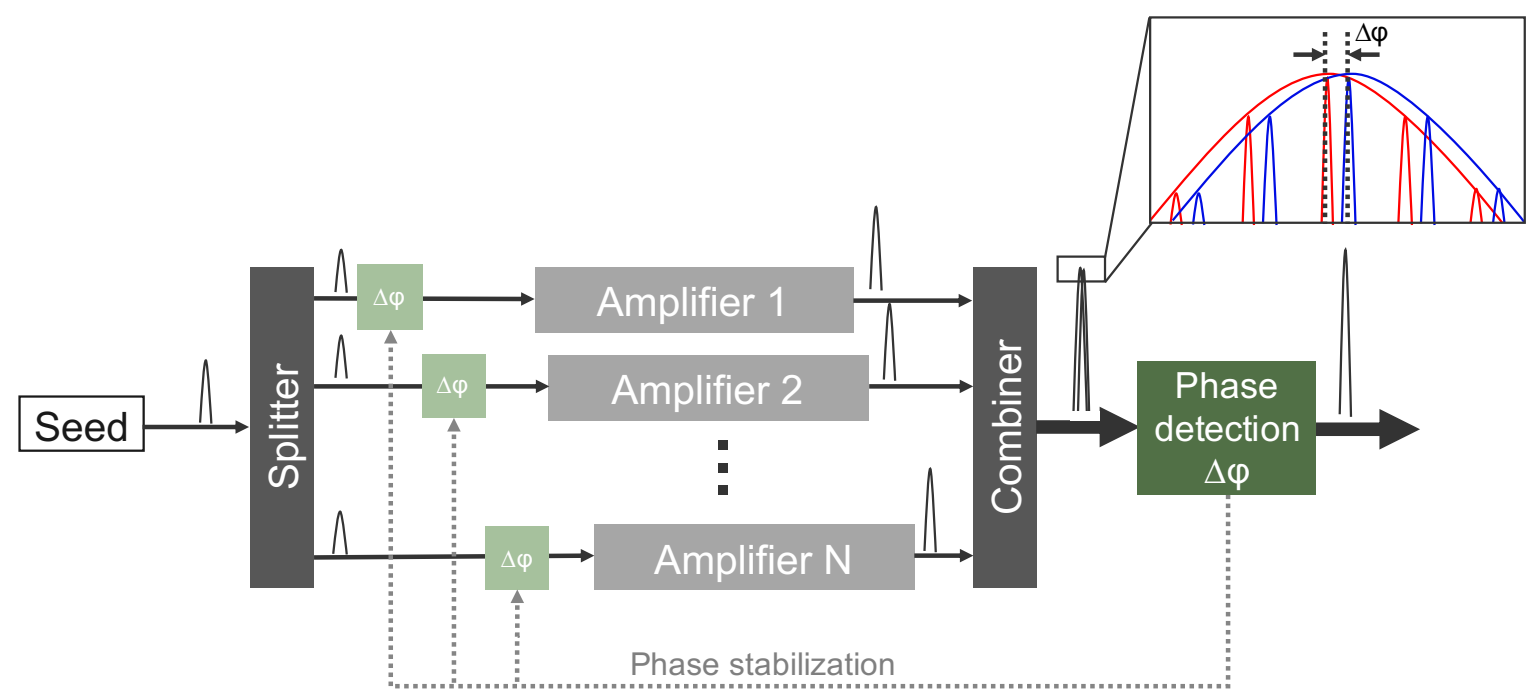

Figure 1. Basic principle of a CBC system.

combiner element is shown. These elements are often used for beam homogenization. ${ }^{8}{ }^{17}$ In a few publications it is described that spatially coherent or nearly spatially coherent light sources cannot be homogenized sufficiently with microlens arrays. ${ }^{18,19}$ With spatially coherent light a spot pattern is generated caused by grating diffraction and interference. It can be understood that such a microlens system can be used as splitter and, with reversing the beam path, as combination element as well.

Furthermore, the pulse repetition rate of ultrafast lasers has been increasing to values over $50 \mathrm{MHz} .{ }^{20}$ For several laser applications such as ablation and marking scanning is a genuine ability usually done electro-mechanically. To exploit the full potential of these laser sources the beam deflection velocity has to be in the order of $1000 \mathrm{rad} / \mathrm{s}$ @1 $1 \mathrm{~m} .{ }^{21}$ Therefore, a highly dynamic control of such laser systems would be of great interest.

For traditional mechanical beam deflection optical lenses or mirror based scanners are used such as piezo scanners or Micro-Electro-Mechanical-Systems (MEMS). Unfortunately, the beam deflection velocities of such systems are typically $100 \mathrm{rad} / \mathrm{s}^{22}$ These systems are fundamentally limited by the mass of the moving parts such as rotating mirrors. For this reason "mass free" technologies, i.e. approaches without moving parts are being developed. This includes Electro-Optical Deflectors (EODs), Acousto-Optical Deflectors (AODs) or Optical-Phased-Arrays (OPAs). EODs and AODs are optical solid state deflectors that relay on the electro-optical respectively acoustooptical effect. For a classical OPA a beam passes an array of liquid crystal phase shifters. ${ }^{23}$ Such systems reach beam deflecting velocity over $\sim 10^{3} \mathrm{rad} / \mathrm{s}^{21}$ But all "mass free" technologies have the common disadvantage that they can only be used at full velocity for low average powers $(\sim \mathrm{mW})$.

One possible solution for this can also be a CBC-system. This will be introduced in the following sections beginning with the theory of MLAs in Sec. 2.1. After this the layout of such a system is discussed in detail in Sec. 2.2 for the one-dimensional (1D) case and in Sec. 2.3 for the two-dimensional (2D) case.

This paper provides a basic description of how to deflect high average powers without reducing the velocity by using CBC. Therefore, a new concept of beam splitting and combining with microlens arrays is presented. In a proof-of-principle experiment a beam splitting and combining system with microlens arrays is shown in which beam deflection and beam shaping with high velocity is possible.

\section{METHODS}

In this section the basics of microlens arrays and beam homogenizers with microlens arrays are introduced. Moreover, the layout of a CBC system is simulated and in a proof-of-principle experiment the beam splitting and beam combination is shown with MLAs. At the end a highly dynamic coherent beam combination based on a setup of microlens arrays is presented and compared with the simulation. 

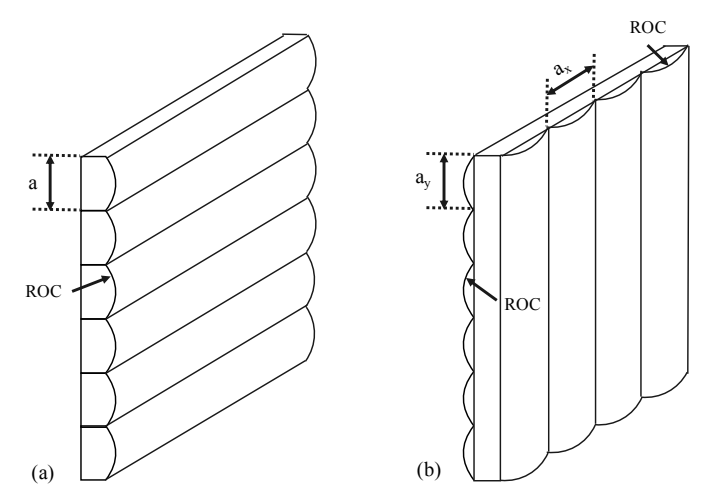

Figure 2. Types of microlens arrays: (a) cylindrical MLA, (b) square MLA

\subsection{Theory of MLAs as splitter and combiner element}

Microlens arrays are already used in certain areas, such as beam homogenization ${ }^{24}$ or for fiber collimation. ${ }^{25}$ In this paper the principle of the imaging homgenizer is the foundation for understanding beam splitting and combination.

The imaging homogenizer is a standard means for beam shaping and consists of two MLAs, mainly square and cylindrical MLAs. In Fig. 2 these two arrays are shown with their characteristics pitch $a$ and radius of curvature $R O C$ which define the effective focal length of a MLA $f_{\mathrm{MLA}}=\frac{R O C}{n-1}$ where $n$ is the refractive index of the lens medium.

The setup for beam combination and beam splitting is based on an imaging homogenizer and is shown in Fig. 3. Generally, the distance between the MLAs is close to $f_{\text {MLA }}$. Subsequently a spherical lens follows, which performs a two dimensional (spatial) Fourier transform (Fourier lens FL). The beam path in Fig. 3 is presented schematically and the intensity in x-z-direction is shown. The intensity in the z-section is adapted. The incident beam illuminates the first MLA and is split into an array of beamlets. The second MLA in combination with the FL acts as an array of objective lenses that superimposes the images of the generated beamlets of the first MLA in the focal plane of the Fourier lens. In geometrical optics a homogeneous intensity profile results. In wave optics, coherence leads to interference and grating diffraction. ${ }^{18,19}$ In conclusion diffraction is caused by the pupil of the microlenses and interference by the periodicity of the array and therefore discrete diffraction orders result according to

$$
\sin (\Theta)=m \cdot \frac{\lambda}{a},(m= \pm 0, \pm 1, \ldots),
$$

leading to individual intensity peaks. In Eq. (1) the grating constant $a$ present the pitch of the MLA. The difference in optical path length creates an interference pattern with the angular period (small-angle approximation)

$$
\Theta=\frac{\lambda}{a}
$$

The angular spectrum is shown in Fig. 4 and within the diffraction angles constructive interference results. As mentioned the beam bundles of the microlenses undergo a mutual path difference $\delta x$ with $\delta x=\Theta \cdot a$. For constructive interference the distance $\delta x$ must be a multiple of the wavelength $\lambda$.

$$
\delta x=\Theta \cdot a=m \cdot \lambda,(m= \pm 0, \pm 1, \ldots) .
$$

Besides the diffraction angle is limited by the numerical aperture $N A$ hence for the bundles with maximum angles $\Theta_{\max }$ applies

$$
\Theta_{\max }=N A=\frac{a}{2 \cdot f_{\mathrm{MLA}}} .
$$

With this theoretical consideration a MLA setup allows a well defined beam splitting process. This means with the choice of the fundamental design parameters of the MLAs an equal power distribution can be achieved. 


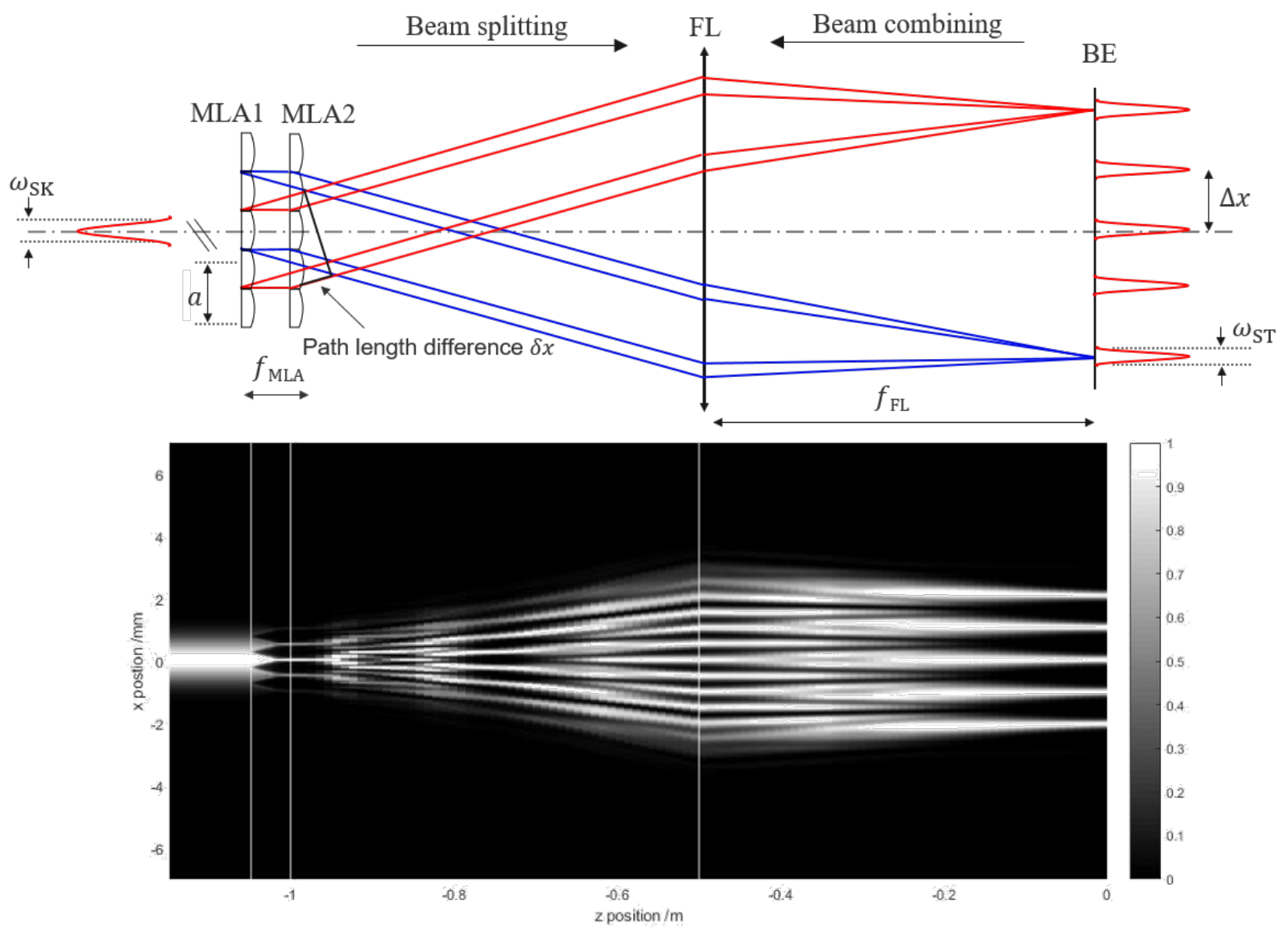

Figure 3. Setup with MLAs as splitter and combiner element (top) and the intensity of the beam path in x-z-direction (bottom) $\left(f_{\mathrm{FL}}\right.$ : effective focal length of the Fourier lens, $F L$ : Fourier lens, $f_{\mathrm{MLA}}$ : effective focal length of the MLA).
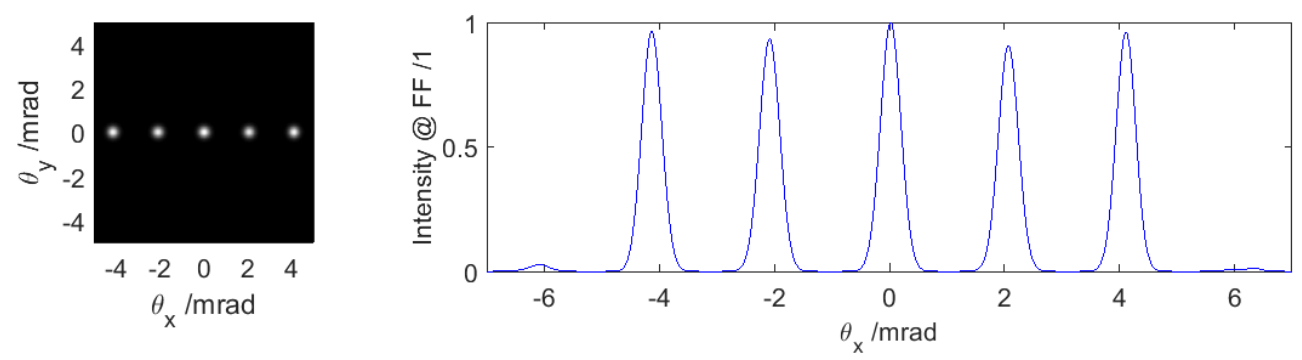

Figure 4. Normalized intensity as a function of the diffraction angle for beam splitting with a pair of MLAs. 
In the case at hand, this heuristically results in

$$
N=\frac{a^{2}}{\lambda \cdot f_{\mathrm{MLA}}}
$$

This has to correspond to the number of generated beams for a homogeneous distribution of the intensity peaks. Accordingly, the design parameters of microlens arrays are selected such that an odd or even number of beams $N$ results. A setup where a well defined beam splitting process is possible should be reversible. For the combining process this means that if $N$ channels should be combined the parameters for the MLAs have to fulfill Eq. (5) to reach a high combining efficiency.

Moreover, for each channel the relative phase must be set to achieve constructive interference. This creates a suitable phase front for the beam combination and ensures a high combination efficiency. Therefore, the angle spectrum of the beam homogenizer illuminated by the wave function $\psi$ is considered

$$
\Psi(\Theta)=\exp \left(\imath \cdot \frac{2 \cdot \pi}{\lambda} \cdot f_{\mathrm{MLA}} \cdot \Theta^{2}\right) \sum_{n} \exp \left(\imath \cdot \frac{2 \cdot \pi}{\lambda} \cdot f_{\mathrm{MLA}} \cdot \frac{\lambda}{a} \cdot n \cdot \Theta\right) \tilde{\psi}\left(\Theta-\frac{\lambda}{a} \cdot n\right)
$$

with $\tilde{\psi}(\Theta) \sim \exp \left(-\frac{\Theta^{2}}{\Theta_{\mathrm{R}}{ }^{2}}\right)$ the divergence $\Theta_{\mathrm{R}}$ of the incident beams. For details see Ref. ${ }^{8}$ The phase factor in Eq. (6) accounts for the difference in optical path between the apertures and, thus for the relative phase results

$$
\delta \varphi_{n}=-\frac{2 \cdot \pi}{\lambda} \cdot f_{\mathrm{MLA}} \cdot \Theta^{2} \cdot n^{21}
$$

with $n$ the position of the individual beam which should be combined. Equation (7) is valid if the beam splitting and beam combining is done with MLAs. These beams (split and combined beams) are defined as diffraction orders. The central spot represents the zero order. After this declaration, variable $n$ is defined accordingly.

Furthermore, with the use of a FL with the focal length $f_{\mathrm{FL}}$ a spacing between the spots in the focal plane follows with

$$
\Delta x=f_{\mathrm{FL}} \cdot \frac{\lambda}{a}
$$

Accordingly, the fill factor $F F$ can be defined as

$$
F F=\frac{2 \cdot \omega_{\text {in }}}{\Delta x}
$$

with $\omega_{\text {in }}$ the beam waist of the individual beams. For the combined beam the beam waist $\omega_{\text {out }}$ depends on the $\mathrm{FL}$ which is used to image the far field with the focal length $f_{\text {FLout }}$ and it follows

$$
\omega_{\text {out }}=\omega_{\text {in }} \cdot \frac{f_{\text {FLout }}}{f_{\mathrm{FL}}}
$$

The beam waist directly at the position of the first MLA $\omega_{\text {MLA }}$ is given by

$$
\omega_{\mathrm{MLA}}=\frac{2 \cdot a}{F F \cdot \pi} .
$$

For beam splitting $\omega_{\text {MLA }}$ presents the size of the input beam and for beam combination it is the beam radius at the second MLA. To characterize the combined output power, the combination efficiency is defined as the power in the desired peak divided by the total output power.

\section{$2.21 \mathrm{D}$ beam splitting and coherent beam combining}

The parameters that are used for the beam splitting and combination process are shown in Tab. 1 . The derived parameters from those are also shown in Tab.1. The experimental setup is depicted in Fig.5. Starting with a mode-locked laser source collimated to a beam waist $\omega_{\text {MLA }}$ at the first MLA (red line in Fig. 5). After this

\footnotetext{
${ }^{1}$ Equation is valid for Fig. 5
} 


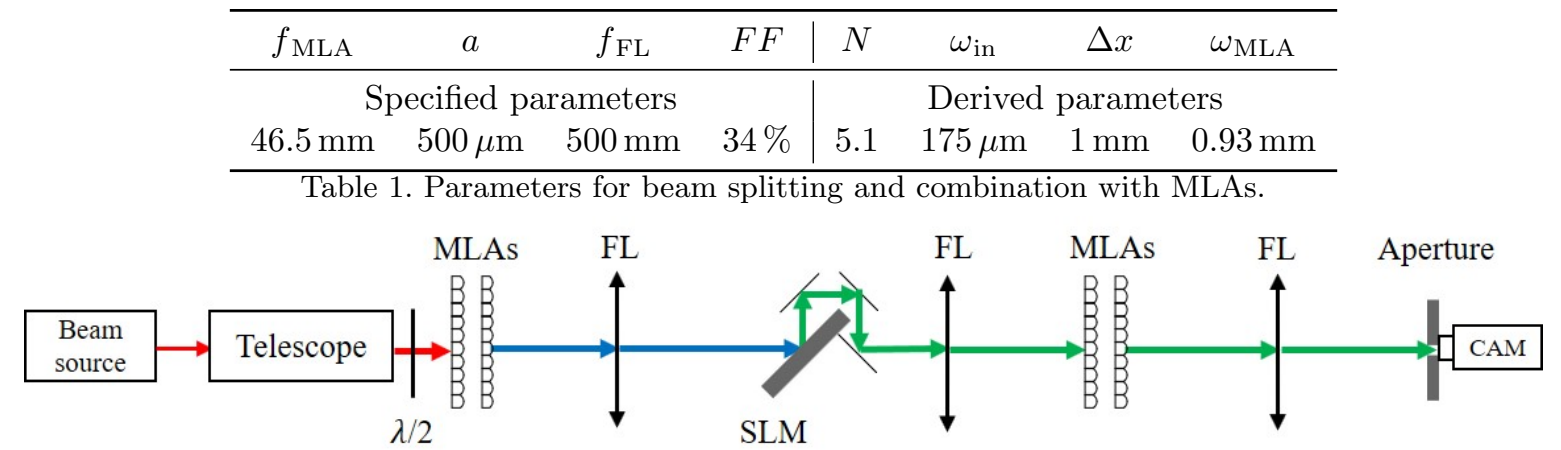

Figure 5. Schematic setup with MLAs as beam splitting and combination element. Red: beam shaping, blue: beam splitting, green: beam combination

the beam splitting (blue line) follows. In the focal plane of the FL an SLM is positioned to compensate the phase differences between the generated beams. It should be noted that alternative elements could be used as phase shifter e.g. a piezo electric actuator with a mirror. Then the combination step (green line) follows with an additional FL to image the far field. At the end, an aperture to determine the resulting combination efficiency is positioned in the focal plane of the last FL.

The experimental results for the beam splitting and combining are shown in Tab.2, which present the power and beam quality of each beamlet and the combined beam. This shows that all beamlets have approximately the same power and beam quality.

\begin{tabular}{ccc}
\hline Beam position & Power $/ \mathrm{mW}$ & Beam quality $M^{2}$ \\
\hline & 1D splitting \\
\hline-2 . Order - 2. Order & $\sim 20$ & $\sim 1$ \\
1D combining & $\sim 91$ & $\sim 1$ \\
\hline \multicolumn{3}{c}{ 2D splitting } \\
\hline-2 . Order x 2. Order & $\sim 4$ & $\sim 1$ \\
2D combining & $\sim 90$ & $\sim 1$ \\
\hline Table 2 . Beam splitting and combining results.
\end{tabular}

A splitting efficiency of $98 \%$ is achieved. The splitting efficiency is defined as the power in the desired N peaks divided by the total output power. In Fig. 6 the red line shows the experimental results and the blue line the simulation. In the simulation as well as in the experiment a slightly difference in the intensity distribution between the peaks is visible. This difference results through the imperfect focal length of the MLA. For this reason analytically 5.1 beams are created with the used MLAs. The next step is the beam combination. Therefore, the relative phase was calculated using Eq. (7) for each beam. It should be noted that the calculated phase a factor of two has to be considered. This is necessary because the first phase difference results for the beam splitting and than again for the beam combination process. The results without phase matching are shown in Fig. 7 with a combination efficiency of about $40 \%$. Through the path length difference which results during the splitting and combination process the beamlets do not interfere constructively. Consequently without phase matching only a low combination efficiency is achieved. However, phase matching leads to a combination efficiency of $91 \%$. Thus, we are slightly below the maximum possible combination efficiency which was calculated by the simulation to $93 \%$. The experimental and simulation results are shown in Fig. 8.

It should be mentioned that the combination efficiency can be even higher. For this experiment a MLA with a focal length of $46.5 \mathrm{~mm}$ is used, which leads analytically to 5.1 beams, according to Eq. (5). Consequently, the combination efficiency reduced. With the assumption that exactly 5 beams are combined a combination efficiency of $98 \%$ is possible according to the simulation. At this point, we should mention that no active phase stabilization is necessary for this particular low power experiment because the beamlets interfere. Therefore, 

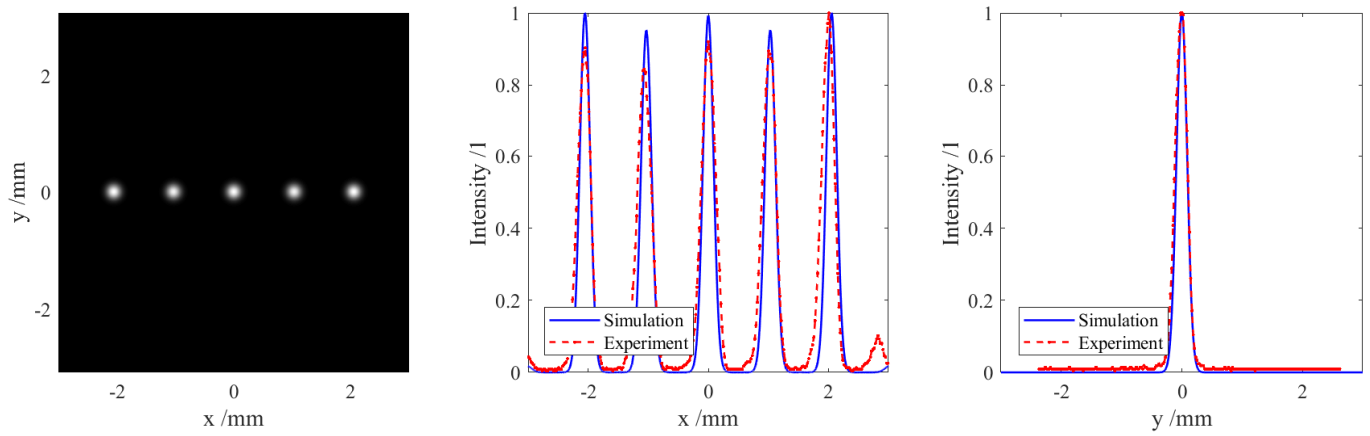

Figure 6. Simulation and experimental results for the 1D beam splitting with MLAs. Left: Image of the generated beamlets. Center: Horizontal cross-section of the beamlets. Right: Vertical cross-section of the beamlets.
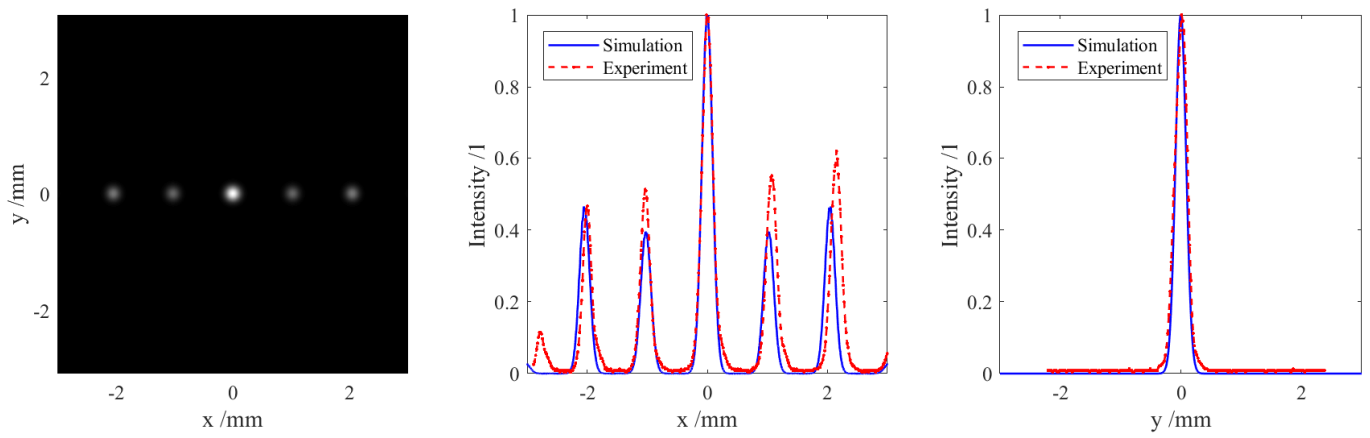

Figure 7. Simulation and experimental results for the 1D combined beam without phase matching. Left: Image of the combined beam. Center: Horizontal cross-section of the combined beam. Right: Vertical cross-section of the combined beam.
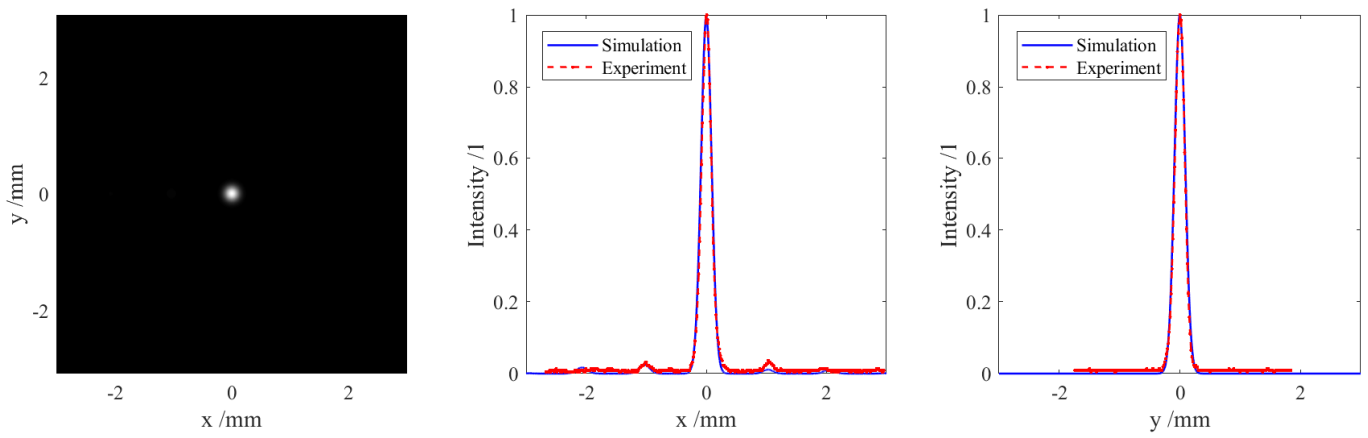

Figure 8. Simulation and experimental results for the 1D combined beam with phase matching. Left: Image of the combined beam. Center: Horizontal cross-section of the combined beam. Right: Vertical cross-section of the combined beam.

all beamlets "see" the same, means that e.g. turbulence's are unlikely to have a significant impact on the combination efficiency. The situation is different with amplifiers, where an active phase stabilization is essential to achieve a high combination efficiency.

\subsection{D beam splitting and coherent beam combining}

In the following the concept is expanded to describe the 2D problem. The same parameters are used as for the $1 \mathrm{D}$ setup but the beam splitting results in a $5 \times 5$ beam matrix, which is presented in Fig. 9. The setup depicted in Fig. 5 is used again but an additional MLA is positioned directly behind the first MLA rotated by 90 degrees with respect to the first one as shown in Fig. 2(b). The experimental and numerical results are in a good agreement. The results for the beam combination without a phase matching are shown in Fig. 10. For this 

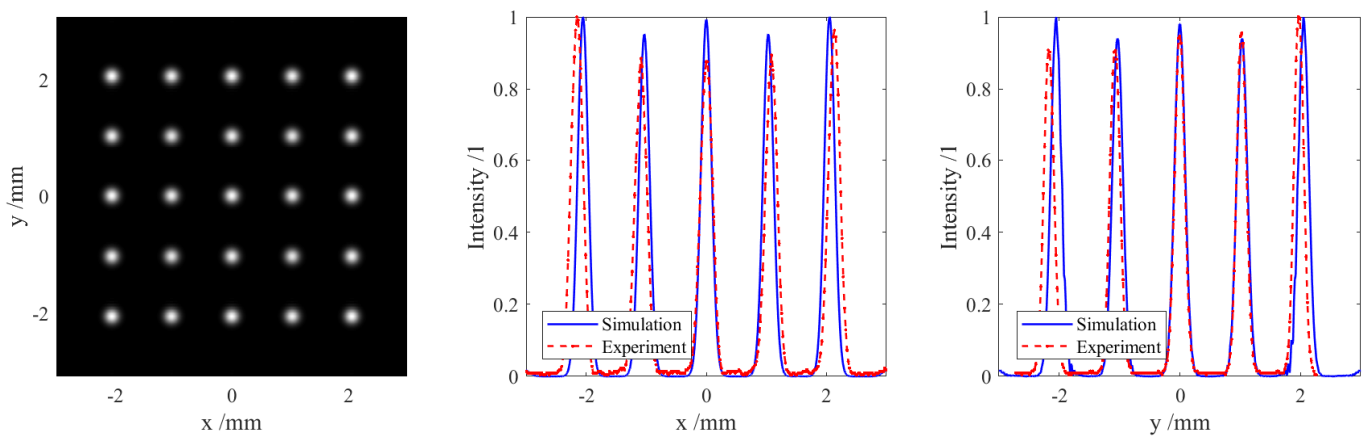

Figure 9. Simulation and experimental results for the 2D beam splitting. Left: Image of the generated beam matrix. Center: Horizontal cross-section of the beam matrix. Right: Vertical cross-section of the beam matrix.
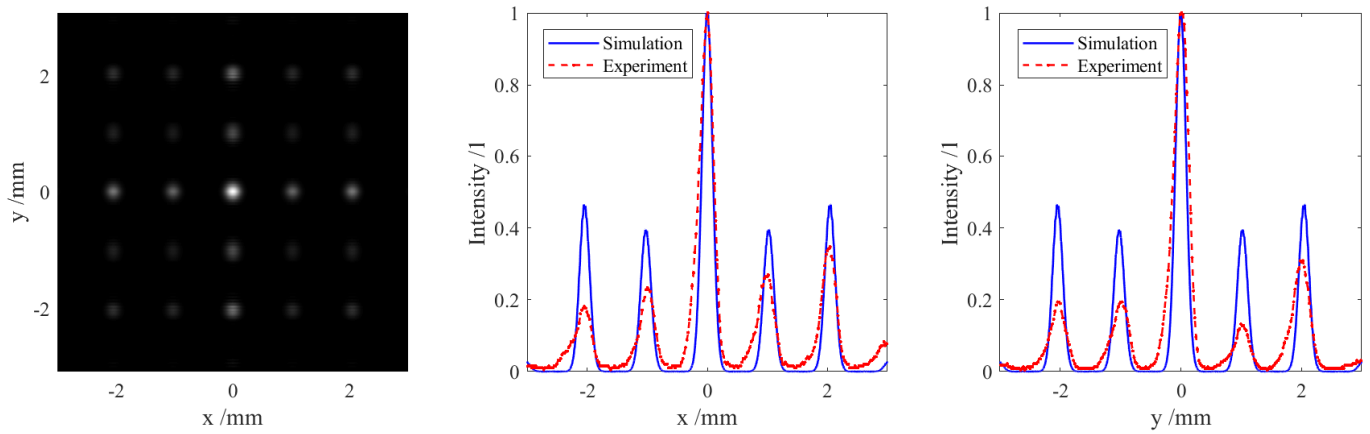

Figure 10. Simulation and experimental results for the 2D beam combination without phase matching. Left: Image of the combined beam. Center: Horizontal cross-section of the combined beam. Right: Vertical cross-section of the combined beam.
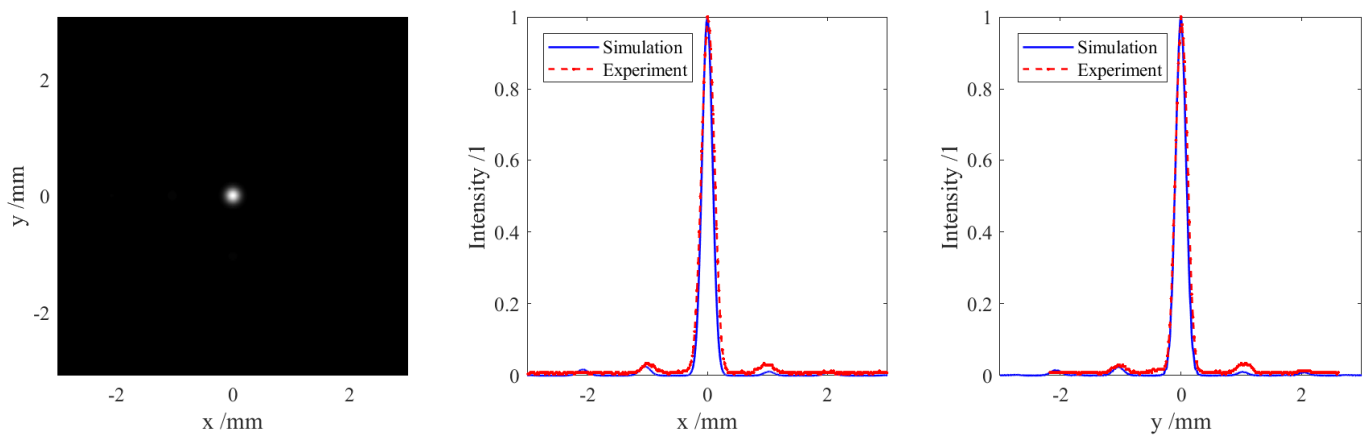

Figure 11. Simulation and experimental results for the 2D beam combination with phase matching. Left: Image of the combined beam. Center: Horizontal cross-section of the combined beam. Right: Vertical cross-section of the combined beam.

a combination efficiency of about $35 \%$ is reached.

The next step is to combine the generated 25 beams. Therefore, Eq. (7) is expanded to a second spatial dimension (y-axis) resulting in

$$
\delta \varphi\left(n_{\mathrm{x}}, n_{\mathrm{y}}\right)=-\frac{2 \cdot \pi}{\lambda} \cdot f_{\mathrm{MLA}} \cdot\left(\Theta_{\mathrm{x}}^{2} \cdot n_{\mathrm{x}}{ }^{2}+\Theta_{\mathrm{y}}{ }^{2} \cdot n_{\mathrm{y}}{ }^{2}\right) \cdot{ }^{2}
$$

The phase differences are again compensated with the SLM and a combination efficiency of $90 \%$ is achieved. Thus, the efficiency of the experiment is close to the theoretical limit, which has been determined with the help of the simulation and is at $93 \%$. The results for the simulation and the experiment are shown in Fig. 11.

\footnotetext{
${ }^{2}$ Equation is valid for Fig. 5
} 

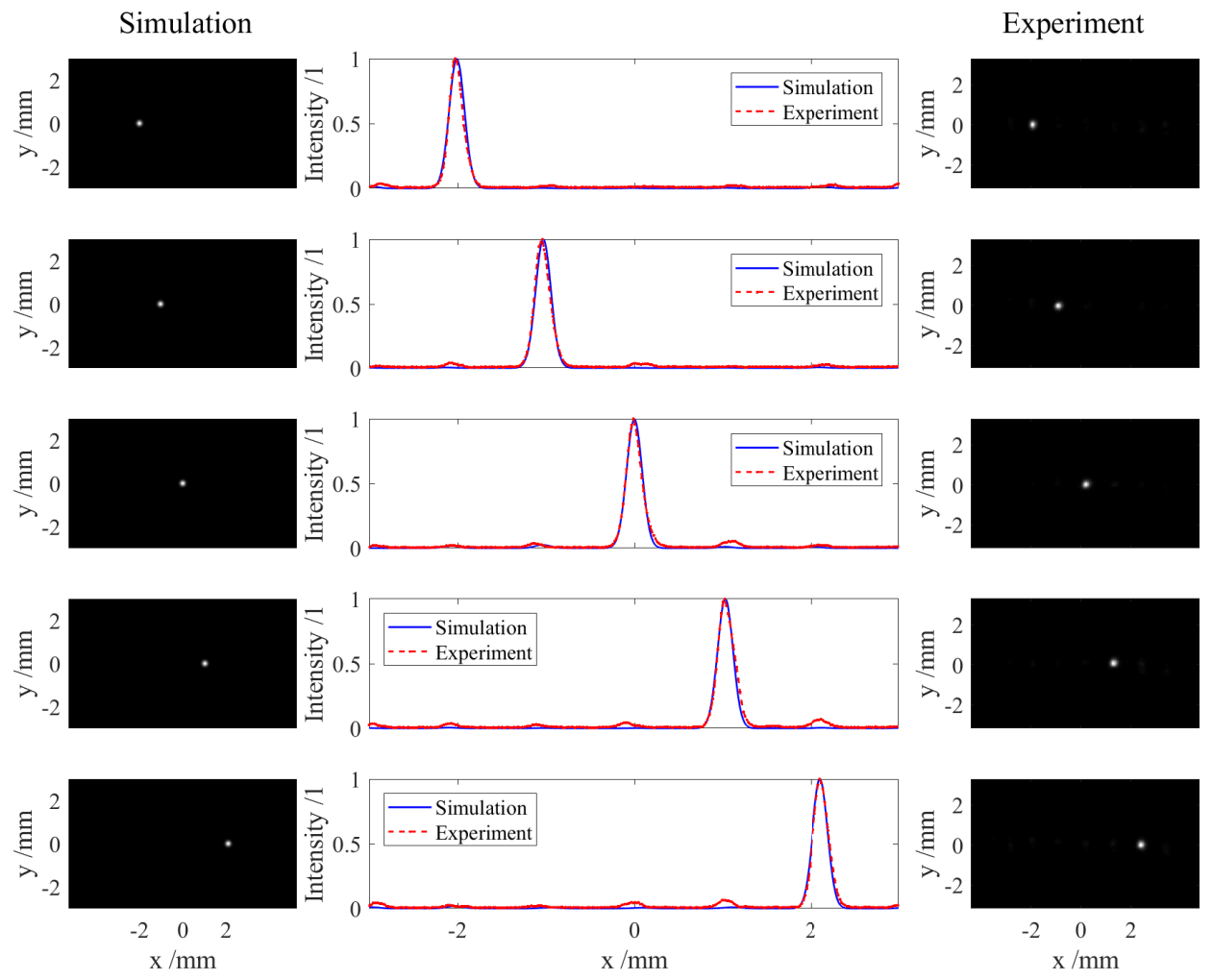

Figure 12. Simulation and experimental results for 1D beam scan. Left: Image of the combined beam with the simulation. Center: Horizontal cross-section of the combined beam. Right: Image of the combined beam with the experiment.

\subsection{Dynamic beam shaping and beam deflection with MLAs}

In Sec.2.2 and 2.3 the splitting and combination of 5 and 25 beams is presented, respectively. In this sections it can be seen that if there is no phase matching undesired diffraction orders result. This hints that with the control of the absolute phase for each beam a beam shaping could be possible. Moreover, a beam combination at a different position than at the zero order should also be possible. For this Eq. (7) is considered. If the beam combination should take place on e.g. the first order, the reference now is the first order. This means that in Eq. (7) $n$ must be increased by one for the first order. For describing this shift an additional therm $m$ is introduced and for Eq. (7) follows

$$
\delta \varphi(n)=\underbrace{-\frac{\pi}{\lambda} \cdot f_{\mathrm{MLA}} \cdot \Theta^{2} \cdot n^{2}}_{\text {phase match for splitting }} \underbrace{-\frac{\pi}{\lambda} \cdot f_{\mathrm{MLA}} \cdot \Theta^{2} \cdot(n+m)^{2}}_{\text {phase match for combination with deflection }}
$$

and for the 2D case it follows with Eq.(12)

$$
\delta \varphi\left(n_{\mathrm{x}}, n_{\mathrm{y}}\right)=\underbrace{-\frac{\pi}{\lambda} \cdot f_{\mathrm{MLA}} \cdot\left(\Theta_{\mathrm{x}}{ }^{2} \cdot n_{\mathrm{x}}{ }^{2}+\Theta_{\mathrm{y}} \cdot n_{\mathrm{y}}{ }^{2}\right)}_{\text {phase match for splitting }} \underbrace{-\frac{\pi}{\lambda} \cdot f_{\mathrm{MLA}} \cdot\left(\Theta_{\mathrm{x}}{ }^{2} \cdot\left(n_{\mathrm{x}}+m_{\mathrm{x}}\right)^{2}+\Theta_{\mathrm{y}}{ }^{2} \cdot\left(n_{\mathrm{y}}+m_{\mathrm{y}}\right)^{2}\right)}_{\text {phase match for combination with deflection }}
$$

The phases are calculated with Eq. (13) respectively Eq. (14) for each diffraction position (-2.Order, -1.Order, 0.Order, 1.Order, 2.Order for $\mathrm{x}$ - and $\mathrm{y}$ - direction). The results for the simulation and experiments with this phases are shown in Fig. 12. Here each combined spot is at a different position and achieved efficiencies are similar to the zero order. This dynamic beam control can only take place on the discrete order positions. That means for five beams there are only five possible positions where the beam can be deflected. Note that the beam 
Simulation
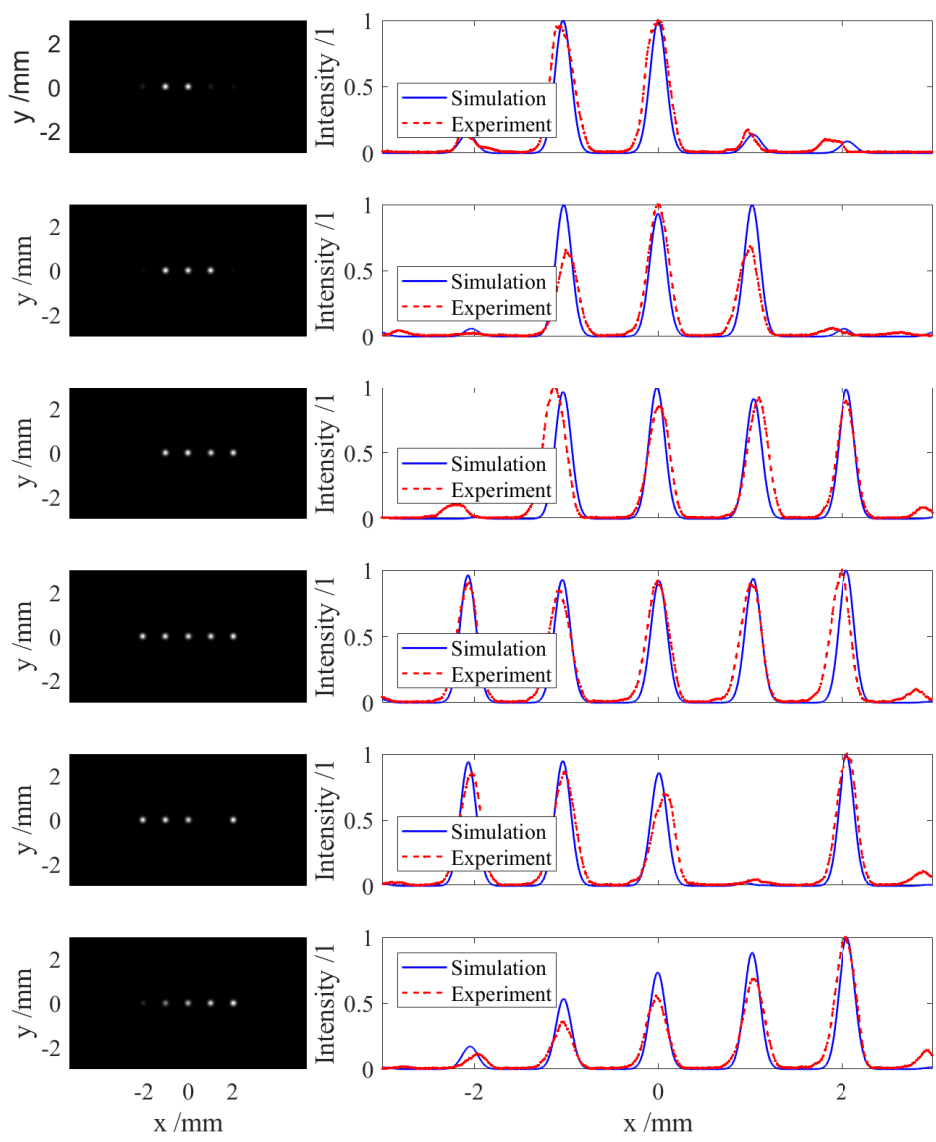

$-2$

$\mathrm{x} / \mathrm{mm}$
Experiment
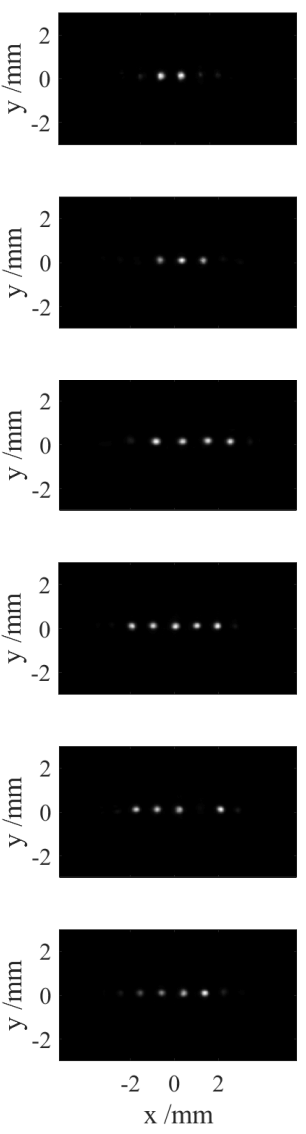

Figure 13. Simulation and experimental results for 1D beam shaping. Left: Image of the combined beam with the simulation. Center: Horizontal cross-section of the combined beam. Right: Image of the combined beam with the experiment.

deflection is discrete, i.e. a position between two different orders does not exist.

After the beam deflection a beam shaping is presented. For this the phases were calculated with a standard min-search-algorithm. Exemplary, the number of combined beams is changed, a single beam is switched off or a power wedge is generated. A power wedge present a linear intensity growth with ascending order. For the power wedge the combined beam will be split into 5 beams with a defined power distribution. For this power wedge, a power distribution of $20 \%$ to $100 \%$ is chosen. Therefore the power distributed as follows: -2 nd order $20 \%$, -1 st order $40 \%$, 0th order $60 \%$, 1st $80 \%$ and 2 nd $100 \%$. The $1 \mathrm{D}$ beam shaping is presented in Fig. 13 . The same principle is for the $2 \mathrm{D}$ case implemented. In Fig. 13 a selection of $2 \mathrm{D}$ beam shaping images are shown.

\section{CONCLUSION}

In conclusion, a novel, compact and simple setup based on standard components for coherent beam combining is presented to combine $N \times N$ beams. Additionally a high dynamic, discrete scanning and beam shaping is implemented. For the combining element a pair of well defined micro-lens arrays is used. In this setup an input beam is split into $5 \times 5$ beams. Subsequently the splitting, the generated 25 beams where combined to one beam again. A combination efficiency of $90 \%$ is achieved. To the best of our knowledge this was the fist time that coherent beam combining with MLAs was presented.

Moreover, with the presented concept it is possible to achieve a dynamic beam combination i.e. the resulting 


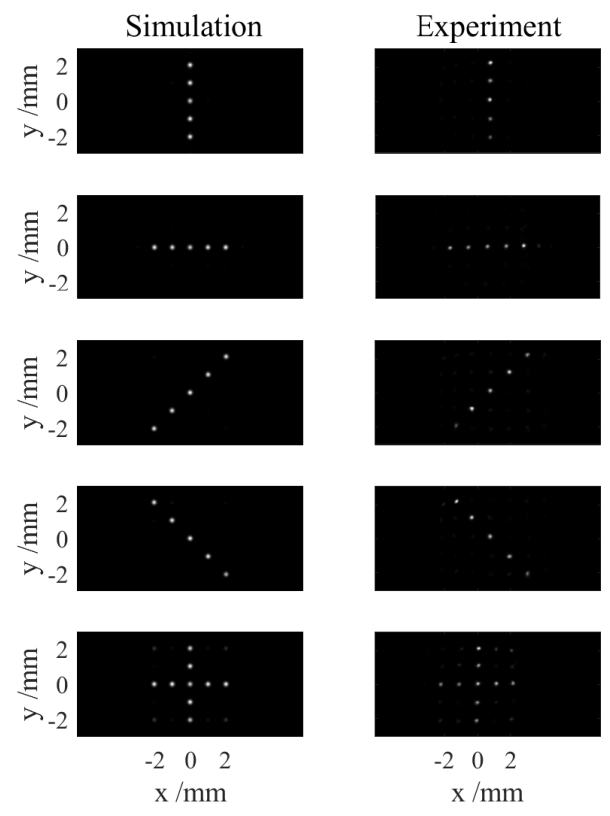

Figure 14. Simulation (left) and experimental (right) results for 2D beam shaping.

number of beams and corresponding positions can be controlled by the absolute phases of the array of input beams. This concept allows switching frequencies in the MHz-range with an EOD or an AOD as phase shifter elements. As a consequence, a beam deflecting velocity over $\sim 10^{6} \mathrm{rad} / \mathrm{s}$ is possible. Moreover, a power modulation of each spot is also possible. For the dynamic beam shaping and deflection there is no significant efficiency loss. Besides, for this proof-of-principle experiment a model is developed and shows excellent agreement with the experiment.

The presented concept shows a new method for highly dynamic beam deflection of high-repetition-rate ultrafast laser systems in the high power regime and offers new possibilities for laser material processing.

\section{REFERENCES}

1. R. Poprawe, H. Weber, and G. Herziger, "Laser applications," 2004.

2. S. J. Augst, T. Fan, and A. Sanchez, "Coherent beam combining and phase noise measurements of ytterbium fiber amplifiers," Optics Letters 29(5), pp. 474-476, 2004.

3. A. Klenke, S. Breitkopf, M. Kienel, T. Gottschall, T. Eidam, S. Hädrich, J. Rothhardt, J. Limpert, and A. Tünnermann, " $530 \mathrm{w}, 1.3 \mathrm{mj}$, four-channel coherently combined femtosecond fiber chirped-pulse amplification system," Optics letters 38(13), pp. 2283-2285, 2013.

4. M. Müller, A. Klenke, A. Steinkopff, H. Stark, A. Tünnermann, and J. Limpert, "3.5 kw coherently combined ultrafast fiber laser," Optics letters 43(24), pp. 6037-6040, 2018.

5. M. Kienel, M. Müller, A. Klenke, J. Limpert, and A. Tünnermann, "12 mj kw-class ultrafast fiber laser system using multidimensional coherent pulse addition," Optics letters 41(14), pp. 3343-3346, 2016.

6. J. Ion, Laser processing of engineering materials: principles, procedure and industrial application, Elsevier, 2005.

7. D. Flamm, D. Grossmann, M. Kaiser, J. Kleiner, M. Kumkar, K. Bergner, and S. Nolte, "Tuning the energy deposition of ultrashort pulses inside transparent materials for laser cutting applications," Proc. LiM 253, 2015.

8. C. Tillkorn, A. Heimes, D. Flamm, S. Dorer, T. Beck, J. Hellstern, F. Marschall, and C. Lingel, "Anamorphic beam shaping for efficient laser homogenization: methods and high power applications," in Laser Resonators, Microresonators, and Beam Control XX, 10518, p. 105181I, International Society for Optics and Photonics, 2018. 
9. D. Flamm, D. G. Grossmann, M. Jenne, F. Zimmermann, J. Kleiner, M. Kaiser, J. Hellstern, C. Tillkorn, and M. Kumkar, "Beam shaping for ultrafast materials processing," in Laser Resonators, Microresonators, and Beam Control XXI, 10904, p. 109041G, International Society for Optics and Photonics, 2019.

10. D. H. Sutter, T. Dietz, D. Bauer, R. Scelle, A. Budnicki, A. Killi, M. Jenne, J. Kleiner, D. Flamm, M. Sailer, et al., "High power and high energy ultrafast disk lasers for industrial applications," in CLEO: Science and Innovations, pp. JM3E-2, Optical Society of America, 2019.

11. T. Hansch and B. Couillaud, "Laser frequency stabilization by polarization spectroscopy of a reflecting reference cavity," Optics communications 35(3), pp. 441-444, 1980.

12. T. M. Shay, "Theory of electronically phased coherent beam combination without a reference beam," Optics Express 14(25), pp. 12188-12195, 2006.

13. M. Vorontsov, G. Carhart, and J. Ricklin, "Adaptive phase-distortion correction based on parallel gradientdescent optimization," Optics letters 22(12), pp. 907-909, 1997.

14. L. Daniault, M. Hanna, L. Lombard, Y. Zaouter, E. Mottay, D. Goular, P. Bourdon, F. Druon, and P. Georges, "Coherent beam combining of two femtosecond fiber chirped-pulse amplifiers," Optics letters 36(5), pp. 621-623, 2011.

15. E. Seise, A. Klenke, J. Limpert, and A. Tünnermann, "Coherent addition of fiber-amplified ultrashort laser pulses," Optics express 18(26), pp. 27827-27835, 2010.

16. A. Klenke, M. Wojdyr, M. Müller, M. Kienel, T. Eidam, H.-J. Otto, F. Stutzki, F. Jansen, J. Limpert, and A. Tünnermann, "Large-pitch multicore fiber for coherent combination of ultrashort pulses," in The European Conference on Lasers and Electro-Optics, p. CJ_1_2, Optical Society of America, 2015.

17. Y. Jin, A. Hassan, and Y. Jiang, "Freeform microlens array homogenizer for excimer laser beam shaping," Optics express 24(22), pp. 24846-24858, 2016.

18. M. Zimmermann, N. Lindlein, R. Voelkel, and K. J. Weible, "Microlens laser beam homogenizer: from theory to application," in Laser Beam Shaping VIII, 6663, p. 666302, International Society for Optics and Photonics, 2007.

19. I. Harder, M. Lano, N. Lindlein, and J. Schwider, "Homogenization and beam shaping with microlens arrays," in Photon Management, 5456, pp. 99-107, International Society for Optics and Photonics, 2004.

20. M. E. Fermann, A. Galvanauskas, and G. Sucha, Ultrafast lasers: technology and applications, vol. 80, CRC Press, 2002.

21. G. Römer and P. Bechtold, "Electro-optic and acousto-optic laser beam scanners," Physics procedia 56, pp. 29-39, 2014.

22. P. Bechtold, R. Hohenstein, and M. Schmidt, "Evaluation of disparate laser beam deflection technologies by means of number and rate of resolvable spots," Optics letters 38(16), pp. 2934-2937, 2013.

23. P. F. McManamon, P. J. Bos, M. J. Escuti, J. Heikenfeld, S. Serati, H. Xie, and E. A. Watson, "A review of phased array steering for narrow-band electrooptical systems," Proceedings of the IEEE 97(6), pp. 10781096, 2009.

24. T. R. Sales, "Random microlens array for optical beam shaping and homogenization," Feb. 22 2005. US Patent 6,859,326.

25. J. Kikuchi, Y. Mizushima, H. Takahashi, and Y. Takeuchi, "Fiber collimator array," Sept. 23 2003. US Patent $6,625,350$. 